\title{
Combined regimens of one year duration in the treatment of multibacillary leprosy-II. Combined regimens with rifampicin administered during 6 months
}

\author{
S R PATTYN, * G GROENEN, $\dagger$ L JANSSENS, $\ddagger$ \\ J DEVERCHIN, $\S$ P GHYS $\dagger$ and the Collaborative Study \\ Group for the treatment of Leprosy \\ * Department of Medical Microbiology, University of Antwerp and \\ Institute of Tropical Medicine, Antwerp, Belgium, $\dagger$ Damien \\ Foundation, Kisangani, Zaire, $\ddagger$ Damien Foundation, Musienene \\ Hospital, Zaire, §Head Foperda Hospital, Pawa, Zaire.
}

\section{Accepted for publication 23 February 1989}

\begin{abstract}
Summary From 1981 to 1983 all multibacillary patients presenting at the collaborating centres in Zaire and Rwanda were treated with one of the following regimens: 6 months supervised daily RMP $600 \mathrm{mg}$, ETH $500 \mathrm{mg}$ and DDS $100 \mathrm{mg}$ or CLO $100 \mathrm{mg}$ followed by 6 months unsupervised daily DDS $100 \mathrm{mg}$ or CLO $100 \mathrm{mg}$ with ETH $500 \mathrm{mg}$ added or not. These regimens gave rise to hepatotoxicity, reversal and erythema nodosum leprosum reactions as described previously.

Bactericidal activity was excellent. Among the 289 patients in the trial, with a mean follow-up period of 3.88 years, no relapses were observed, with an upper $95 \%$ confidence limit of 0.35 per 100 person years.

Because of the hepatotoxicity, alternative short-course therapies need to be tested.
\end{abstract}

\section{Introduction}

In the accompanying paper ${ }^{1}$ it is shown that multibacillary (MB) leprosy patients can be successfully treated with a combined regimen of one year duration with rifampicin (RMP) administered during one year. One of the drawbacks of this regimen is the logistic problem created by the need to keep to patients under close supervision for 12 months, of ten necessitating hospitalization. The application in the field of combined regimens could be considerably simplified if the supervised phase could be shortened to 6 months. Therefore a prospective therapeutic trial was organized in Zaire and Rwanda, studying regimens of one year duration but in which RMP was administered during the first 6 months only. The results of the patient cohorts taken into the trial in 1980, 1981, 1982 and 1983 who have been followed up to 5 years after the end of treatment are presented in this analysis.

Tl Collaborative Study Group: Zaire, N Bossaer, L Breugelmans, L Coussens, R De Raedt, E Nollet, A M Passagez, J Verlinden; Rwanda, R Boen, E Schacht. 


\section{Patients and methods}

Diagnostic procedures and criteria for evaluation are identical to the 12 month RMP trial, and are detailed in the accompanying paper. ${ }^{1}$ However, in Zaire and R wanda biopsies were taken routinely. Thus all patients were thoroughly evaluated clinically, neurologically, bacteriologically, as well as histopathologically, at the moment of intake and at each annual follow-up examination. All results were entered on specially designed files, a copy of which was sent to Antwerp.

Multibacillary (MB) leprosy was diagnosed when the bacteriological index (BI) was 2 or more (Ridley Scale ${ }^{2}$ ) at any of the 3 sites examined (1 earlobe, 2 skin lesions), or in the skin biopsy. Patients were taken into the trial if they agreed to stay in or near the treatment centre for 6 months, or if they were capable of coming to the treatment centre daily. Thus the RMP phase of the therapy was fully supervised. The second phase of the therapy, 6 months without RMP, was unsupervised. Every month the patient came to the treatment centre to collect his monthly provision of ethionamide (ETH) and dapsone (DDS) or clofazimine (CLO). Patients were thus seen daily during the first 6 months of therapy, at least once a month during the next 6 months of therapy, and after stopping treatment at least once a year for the annual follow-up examination. Male patients never treated before or treated previously for less than 5 years with DDS were randomly allocated to one of the following two regimens:

RED/D: daily (except Sundays) supervised RMP 600 mg ETH 500 mg DDS 100 mg during 6 months, followed for 6 months by daily, unsupervised DDS $100 \mathrm{mg}$.

RED/ED: identical with RED/D during the first 6 months, but followed for 6 months by daily, unsupervised ETH $500 \mathrm{mg}$ and DDS $100 \mathrm{mg}$.

All female patients, and male patients treated previously during 5 years or more with DDS were randomly allocated to one of the following regimens:

REC/C: identical with RED/D but DDS replaced by CLO $100 \mathrm{mg}$.

REC/EC: identical with RED/ED but DDS replaced by CLO $100 \mathrm{mg}$.

No other antileprosy drug than DDS had been available previously in these regions.

DDS was replaced by CLO in females because of the supposed protective action of CLO against ENL reactions that cannot be treated with thalidomide in women of child-bearing age, and in patients treated previously for 5 years or more because resistance to DDS could be suspected in those patients. Every drug administration or delivery, as well as the occurrence of complications (mainly reactions and hepatitis) and their treatment, was noted on a treatment file. Reactions were treated, ${ }^{3}$ but without using CLO, to avoid interference with the trial results by the leprostatic action of CLO. Hepatitis was diagnosed mainly on clinical grounds, since most centres participating in the trial did not have facilities to determine SGOT, SGPT and serum bilirubin. If a diagnosis of hepatitis was made, RMP and ETH were stopped and the patient was continued on DDS or CLO.

Statistical analysis was done by the Student $t$-test, the $\chi^{2}$ test with Yates' continuity correction for $2 \times 2$ tables, and the Mantel-Haenszel $\chi^{2}$ test for comparing rates. Confidence limits, assuming a binomial distribution, were taken from Tabulae Scientificae. ${ }^{4}$

\section{Results}

Three hundred and fifty-six patients were started on one of the trial regimens in 1980, 1981, 1982 and 1983. Before the first year of follow-up, 4 died of liver complications, 17 died of unrelated causes, and $43(12 \cdot 1 \%)$ were lost to follow-up. Three patients who took leprostatic drugs after the end of the study regimen were eliminated from the study, leaving 289 patients for analysis. As shown 
Table 1. Annual intake and follow-up of patients

\begin{tabular}{lrrrrrrrrr}
\hline & 1981 & 1982 & 1983 & 1984 & 1985 & 1986 & 1987 & 1988 \\
\cline { 2 - 10 } Intake & 71 & 98 & 120 & & & & & \\
\hline Died & - & - & - & 1 & 3 & 5 & 4 & 1 \\
Lost & - & - & - & 1 & 2 & 15 & 29 & - \\
Patients seen & - & - & 71 & 167 & 282 & 262 & 229 & 110 \\
Patients not yet seen & & & & & & & & 118 \\
\hline
\end{tabular}

Table 2. Annual decrease of the bacteriological index after stopping treatment

\begin{tabular}{lrc}
\hline Number of Patients & \multicolumn{2}{l}{289} \\
\hline Annual decrease of BI in units & $n^{*}$ & $\%$ \\
\hline 0 & 3 & 1 \\
$0 \cdot 01-0 \cdot 49$ & 23 & 8 \\
$0 \cdot 50-0 \cdot 99$ & 125 & $43 \cdot 7$ \\
$1 \cdot 00-1 \cdot 49$ & 86 & $30 \cdot 1$ \\
$1 \cdot 50$ and more & 49 & $17 \cdot 1$ \\
\hline Mean & & $1 \cdot 01$ \\
Negative a t moment of analysis & 205 & $(71 \cdot 7 \%)$ \\
\hline
\end{tabular}

* Data for 3 patients incomplete.

in Table 1, 13 patients were followed for 1 year, after the end of treatment, 24 for 2 years, 64 for 3 years, 100 for 4 years, 60 for 5 years and 28 for 6 years, or a total of 1121 person years of follow-up. The mean duration of follow-up was 3.88 years. Previously treated patients were followed for a longer period than previously untreated ( 4.03 vs 3.64 years), and patients receiving CLO were followed for a longer period than patients receiving DDS (4.04 vs 3.55 years). These differences are statistically significant $(p<0 \cdot 0025)$.

The bacteriological characteristics of the patients at the moment of intake were comparable in all groups. The BI at intake* ranged from 0.5 to 6 , with a mean of 3.54 (standard deviation 1.14) and a median of $3 \cdot 5$.

Clinically all patients improved rapidly, and continued to do so after the end of therapy. The BI continued to decrease af ter the end of treatment in almost all patients. The annual decrease for 286 patients ( 3 files were incomplete) is summarized in Table 2 . the mean annual decrease in all patients was 1.01 units/year. There were no differences between mean decreases in each group that were significant at the $5 \%$ level. In 3 patients the BI did not decrease, but they could be followed for only a relatively short time: 1 patient was lost after 1 year, two others died after 1 and 2 years respectively. In 4 patients the BI did not decrease as expected. In patient A from 5 to 3.5 in 5 years, in patient $B$ from 3 to 2.5 in 4 years, in patient $C$ from 5.5 to 4.5 in 5 years, in patient $D$ from 5 to 4.5 in 5 years. These 4 patients show a satisfactory clinical and histopathological evolution (all bacilli are extremely granular). In the biopsies the macrophage granulomas in most cases persisted, they contained very granular bacilli and finally became empty.

* Calculations are based on mean BI per patient, rounded to the nearest $-\cdot 5$. Thus a skin smear result of 4-2-2 becomes $2 \cdot 5$, while a result of $2-0-0$ becomes $0 \cdot 5$. 
In total, 9 patients died while still bacteriologically positive. Of the remaining $277,205(74 \%)$ have become bacteriologically negative at the moment of the present analysis. No differences between any of the groups are manifest.

No relapses were seen in any of the patients (a relapse is defined clinically by new lesions or aggravation of existing lesions, and bacteriologically by a BI becoming positive again after negative, or increasing by at least 2 units at any site). The $95 \%$ confidence intervals for 0 relapses according to the person years per year of follow-up are given in Table 3 , and reaches at present between 0 and $0 \cdot 35$ per 100 person years.

Eleven patients presented a reactivation of existing lesions after the end of treatment. This reactivation was not accompanied by an increase in BI and responded well to anti-inflammatory treatment. Four of the reactivations were biopsied: 3 showed a BT image, and one showed an aspecific perivascular cellular infiltrate. These reactivations are late reversal reactions. Their incubation time reaches from 2 to 50 months after the end of treatment, with a mean of 22.5 months and a median of 24 months.

As described earlier, ${ }^{5}$ hepatotoxicity occurred in $5 \%$ of patients receiving the regimens under study. No differences between any of the regimens were manifest. Upgrading and ENL reactions, as well as the neurological evolution, were described in detailf or two of the centres participating in the trial. ${ }^{3}$ The situation is comparable in the other participating centres.

Compared with the situation at thestart, $15 \cdot 1 \%$ of the patients at the last follow-up examination showed an increase and $54.5 \%$ a decrease in the number of hypertrophied nerves.

Previously untreated patients showed a significantly higher increase in the number of hypertrophied nerves $(23 \cdot 1 \%)$ than previously treated patients $(9 \cdot 7 \%)(0 \cdot 005<p>0 \cdot 001)$. Previously treated patients receiving DDS also showed a significantly higher increase of nerve hypertrophy than previously treated patients receiving CLO $(26 \cdot 3 \%$ vs $7 \cdot 7 \%, 0.05<p>0.025)$. However, patients receiving DDS had by definition less than 5 years of previous treatment, whereas patients receiving CLO had by definition more than 5 years previous treatment. Whether CLO in itself protects the nerves cannot be concluded from this study.

Mutilations, coded by the WHO disability score ${ }^{6,7}$ deteriorated in $80(27.4 \%)$ patients and improved in $96(32.9 \%)$. No difference in deterioration is manifest between patients receiving DDS or CLO, nor between previously treated and previously untreated patients.

\section{Discussion}

Antibacterial regimens in MB leprosy should be evaluated on clinical and bacteriological grounds, and on the incidence of relapses after treatment. The results presented here show that MB leprosy can be successf ully treated with regimens of 1 year duration, and that RMP administration can be

Table 3. Ninety five per cent confidence intervals for 0 relapses, according to the person years of follow-up, and assuming a binomedial distribution of the occurrence of relapses

\begin{tabular}{|c|c|c|c|}
\hline $\begin{array}{l}\text { Years of } \\
\text { follow-up }\end{array}$ & $\begin{array}{l}\text { Number of } \\
\text { patients seen }\end{array}$ & $\begin{array}{l}\text { Person years } \\
\text { of follow-up } \\
\text { (cumulative) }\end{array}$ & $\begin{array}{l}\text { Confidence interval } \\
\text { for } 0 \text { relapses per } \\
100 \text { person years }\end{array}$ \\
\hline 1 & 289 & 289 & $0-1 \cdot 27$ \\
\hline 2 & 276 & 565 & $0-0.66$ \\
\hline 3 & 252 & 817 & $0-0.45$ \\
\hline 4 & 188 & 1005 & $0-0.37$ \\
\hline 5 & 88 & 1093 & $0-0.35$ \\
\hline 6 & 28 & 1121 & $0-0.35$ \\
\hline
\end{tabular}


limited to the first 6 months, which confirms the observations made in a smaller previous study. ${ }^{8}$ All patients improved clinically, and the BI continued to decrease after therapy ended in $98.9 \%$ of the patients. The mean annual decrease of the $\mathrm{BI}(1.01)$ is comparable to the decrease observed af ter the 8-44 RED $(C)$ regimen discussed in the accompanying paper. ${ }^{1}$ As was the case there, slow $(0 \cdot 01-0 \cdot 49$ units per year) and rapid (more than 1.50 units per year) decreasers are observed unrelated to the BI at intake.

The mean follow-up period for the 8-44 $\operatorname{RED}(C)$ study ${ }^{1}$ is longer than for the present study: $4 \cdot 29$ vs 3.88 years. There are more patients negative at the moment of analysis after $8-44 \operatorname{RED}(C)$ than after the present regimens $(87 \cdot 7 \%$ vs $74 \%)$. However, this may be due to the higher BI at intake in the present study (3.54 vs 3.13) and the shorter period of follow-up. The reasons previously treated patients attended follow-up examinations more regularly and for longer than previously untreated ones may be the result of a long-standing habit.

No relapses were observed in any of the patients. Since they were followed for a total of 1121 person years, with a mean follow-up of 3.88 years, we may state that the regimens in the present study are expected to give rise to less than 0.35 early relapses per 100 person years, $95 \%$ of the time.

The present results are comparable to the 0 relapses observed after $8-44 \operatorname{RED}(C)$ in the accompanying paper. ${ }^{1}$ They are significantly better than the results obtained after short-course DDS monotherapy: 5 relapses for 22 person years of follow-up; $;^{10} 6$ relapses for $20 \cdot 2$ person years of follow-up; ${ }^{11}$ and 15 relapses for 255 person years of follow-up ${ }^{12}$ (all: $p<0 \cdot 0005$ ). Compared with the relapses observed after prolonged DDS monotherapy ${ }^{13}$ ( 11 relapses for 1297 person years of followup) the present regimens are also significantly better $(p<0 \cdot 001)$.

The increase in nerve hypertrophy, as well as the deterioration of the mutilations in the present study (results not shown) are comparable to the results found in the accompanying paper ${ }^{1}$ af ter 8-44 $\mathrm{RED}(\mathrm{C})$ in Burundi patients.

The present study suggests that it is possible to treat MB leprosy successfully with a 1 year combined regimen with RMP administered during the first 6 months. The 4 regimens under study are comparable in terms of clinical improvement, mean bacteriological decrease and absence of relapses. The addition of ETH during the second 6 months of therapy does not improve any of the results.

The hepatotoxicity of the regimens under study is unacceptable, the more so, since it was only detected on clinical ground. These regimens were therefore abandoned in February 1984. The search for equally successful, but less toxic regimens, easily applicable in difficult field situations, needs to be continued.

\section{References}

1 Pattyn SR, Bourland J, Grillone S, Groenen G. Combined regimens of one year duration in treatment of multibacillary leprosy. I. Combined regimens with rifampicin administered during one year. Lepr Rev. $1989 ; 60$.

2 Ridley S. The histopathology of leprosy. Documenta Geigy, Basel, Switzerland.

${ }^{3}$ Groenen G, Janssens L, Kayembe T, Nollet E, Coussens L, Pattyn SR. A prospective study on the relationship between intensive bactericidal therapy and leprosy reactions. Int J Lepr, 1986; 54: $236-44$.

4 Tabulae Scientificae. Documenta Geigy. 7th Ed. 1978 Ciba-Geigy. Basel, Switzerland.

${ }^{5}$ Pattyn SR, Janssens L, Bourland J, Saylan T, Davies E, Grillone S, Ferracci C. Hepatotoxicity of the combination of rif ampicin-ethionamide in the treatment of multibacillary leprosy. Int J Lepr, 1984; 52: $1-6$.

6 WHO Study Group Expert Committee on Leprosy. Third Report. Technical Report Series No. 319. WHO: Geneva 1966.

7 WHO Expert Committee on Leprosy. Fourth Report. Technical Report Series 459. WHO: Geneva 1970.

8 Pattyn SR, Saint André P, Ferracci C, Baquillon G. Comparative study of two regimens of combined therapy of one year duration in multibacillary leprosy. Int Lepr, 1984; 52: 297-303.

9 Pattyn SR, Groenen G, Bourland J, Grillone S, Grossettê G, Husser JA, Janssens L. The incubation time of 
relapses after treatment of multibacillary leprosy with dapsone monotherapy and rifampicin containing regimens. Europ J. Epidemiol, 1988; 4: 231-34.

10 Erickson PT. Relapse following apparent arrest of leprosy by sulf one therapy. Int J Lepr, 1952; 20: 229-38.

11 Price RB. Relapse of leprosy in American Samoa. Am J Trop Med Hyg 1959; 8: 358-63.

12 Lowe J. The late results of sulphone treatment of leprosy in East Nigeria. Lepr Rev, 1954; 25: 113-24.

13 Waters MFR, Rees RJW, Laing ABG, Khoo Kah Fah, Meade TW, Parikshak N, North WRS. The late of relapse in lepromatous leprosy following completion of twenty years of supervised sulphone therapy. Lepr Rev, 1986; 57: 101-9. 